\title{
Therapeutic Effect of His-Purkinje System Pacing Proportion on Persistent Atrial Fibrillation Patients With Heart Failure
}

\author{
Fei Tong ${ }^{1}$ and Zhijun Sun $^{1}$ \\ ${ }^{1}$ Shengjing Hospital of China Medical University
}

November 16, 2021

\begin{abstract}
Background His-Purkinje system pacing(HPSP) combined with atrioventricular node (AVN) ablation is an effective therapy for atrial fibrillation (AF) patients with heart failure (HF).However, AVN ablation is accompanied with some limitations and disadvantages. HPSP combined with $\beta$-blocker reduces inherent heart rate and increases pacing ratio, which may be an alternative to HPSP combined with AVN ablation. This study was to assess the therapeutic effect of different His-Purkinje system pacing proportions on AF patients with HF. Methods The study enrolled 30 consecutive persistent AF patients with HF who underwent HPSP. Heart rate was controlled by medical therapy. New York Heart Association (NYHA) classification, serum NT-proBNP concentration, echocardiographic parameters were assessed at each follow-up. Results The best cut-off value of pacing proportion to predict MACE by ROC analysis was 71\%. In high pacing proportion group, there were significant improvements of NYHA classification, NT-proBNP concentration, LVEF and LVEDD from the baseline in wide QRS complex patients and HFrEF patients, and there were significant improvements in NYHA classification, NT-proBNP concentration from baseline in narrow QRS complex patients and HFpEF patients, moderate but no significant improvements of LVEF and LVEDD were observed in those patients groups. In low pacing proportion group, there were no significant improvements of NT-proBNP concentration, LVEDD or LVEF regardless of baseline QRS duration or LVEF ( $\mathrm{P}>0.05)$. Conclusion High pacing proportion of HPSP has a beneficial effect on the prognosis of persistent AF patients with heart failure.
\end{abstract}

Therapeutic Effect of His-Purkinje System Pacing Proportion on Persistent Atrial Fibrillation Patients With Heart Failure

Fei Tong

Department of Cardiology, Shengjing Hospital of China Medical University, Shenyang, China

Correspondence

Zhijun Sun, Department of Cardiology Shengjing Hospital of China Medical University, No.36, Sanhao Street, Heping District, Shenyang 110004, China.

Funding: None

Conflict of Interest:None

Atrial fibrillation $(\mathrm{AF})$ is the most common arrhythmia in patients with heart failure $(\mathrm{HF})^{1,2}$. $\mathrm{AF}$ and $\mathrm{HF}$ have similar risks and mechanisms ${ }^{3}$ related to physiological processes that initiate and sustain each other ${ }^{4}$. Current methods to control heart rate and rhythm in patients with atrial fibrillation include drug therapy, radiofrequency ablation and cryoablation, but drug therapy is sometimes ineffective and may be accompanied by adverse reactions. Catheter ablation has a high recurrence rate of $\mathrm{AF}^{5}$, especially the recurrence rate of persistent atrial fibrillation can reach up to $50 \% \%^{6,7}$. AF duration for more than 2 years and heart failure are 
identified as predictor for $\mathrm{AF}$ recurrence ${ }^{6,8}$. Therefore, the clinical treatment of persistent atrial fibrillation with long duration and heart failure is still challenging.

His-Purkinje system pacing (HPSP) includes His bundle pacing (HBP) and left bundle branch pacing (LBP). It can deliver physiological activation and avoid ventricular and intraventricular asynchrony contraction via intrinsic conduction pathway, hence it can improve the hemodynamic performance of patients with heart failure. In 2000, Deshmukh et al. first performed HBP and atrioventricular node (AVN) ablation in patients with persistent $\mathrm{AF}$, dilated cardiomyopathy and heart failure with reduced ejection fraction (HFrEF), and improvement of left ventricle dimensions and cardiac function were observed ${ }^{9}$. In 2017, Huang et al. implemented HBP and AVN ablation in heart failure patients with persistent AF combined with HFrEF or heart failure with perserved ejection fraction (HFpEF), and observed improvement in symptoms and echocardiographic parameters ${ }^{10}$. However, after AVN ablation, HBP pacing threshold increased by $0.5 \mathrm{~V}$ to $1.5 \mathrm{~V}^{11}$. AVN ablation artificially causes complete atrioventricular block and pacer-dependence, and physiology of HPSP is also different from intrinsic conduction system. Therefore, clinically physicians can prescribe $\beta$-blockers for patients with persistent AF and HF to inhibit AVN conduction function and reduce intrinsic heart rate, so as to achieve a high proportion of HPSP and the purpose of rate and rhythm control. However, there are few studies on this therapy. This study aimed to assess the therapeutic effect of different His-Purkinje system pacing proportion on persistent AF patients with HF.

\section{Study Patients}

Consecutive patients who met the inclusion criteria were enrolled between October 2017 and July 2020. The inclusion criteria were the following: (1) Persistent AF with bradycardia or long RR interval, or AF recurrence after radiofrequency ablation, or unsuitable for radiofrequency ablation; (2) Heart failure in New York Heart Association (NYHA) classification was referred to II - IV class; (3) Patients were at least 18 years old and not pregnant.

Patients with any of the following conditions were excluded: (1) Severe mitral or aortic valve stenosis or regurgitation; (2) Congenital heart disease requiring cardiac surgery; (3) Severe chronic obstructive pulmonary disease; (4) Chronic kidney disease requiring long-term dialysis. All patients were provided written informed consent.

\section{Implantation Procedure}

His bundle pacing: Delivery sheath (model C315His, Medtronic, Inc ) was sent to the right atrium or right ventricle through 0.035 guide wire via subclavian vein or axillary vein. The Select Secure lead (model 3830, Medtronic, Inc) was navigated into the vicinity of His bundle through delivery sheath. During the lead placement procedure, the 12-lead ECG and intracardiac electrogram via pacing lead were monitored and recorded. After His bundle potential was identified, 12 lead electrocardiograms were recorded continuously by high pressure pacing method with higher than normal heart rate. Through synchronous ECG, we could determine whether His bundle is captured. After the ideal position was determined, the pacemaker lead was vertically stapled into the room interval (IVS) to maintain the stability of the sheath. Pacing threshold, sensed R-wave amplitude and lead impedance were measured. The morphology of ECG at different output voltages was recorded. Thresholds of selective and non-selective His bundle capture were recorded. Nonselective His bundle pacing was the first choice in our center, and the acceptable threshold was[?]2V / 0.5ms. If parameters of His bundle pacing were not acceptable, left bundle branch pacing or ventricular backup pacing would be attempted. The lower rate for HBP was initially set at 70 beats $/ \mathrm{min}$.

Selective His bundle pacing (S-HBP) criteria: (1) The paced QRS duration and morphology are both identical to intrinsic QRS; (2) The pacing stimulus to QRS onset interval (PV interval) is identical to His-QRS onset interval (H-V interval); (3) The potential of the His bundle can be determined by a narrow QRS at low output pacing and the presence of QRS broadening at high output pacing; (4) Pacing signal can be seen from the beginning of QRS complex.

Non-selective His bundle pacing (NS-HBP) criteria: (1) P-V interval is less than or equal to H-V interval; (2) 
The potential of the His bundle can be identified, with QRS widening at low output pacing and narrowing at capture of His bundle; (3) A pseudo pre-excitation wave can be immediately after His-pacing stimulus.
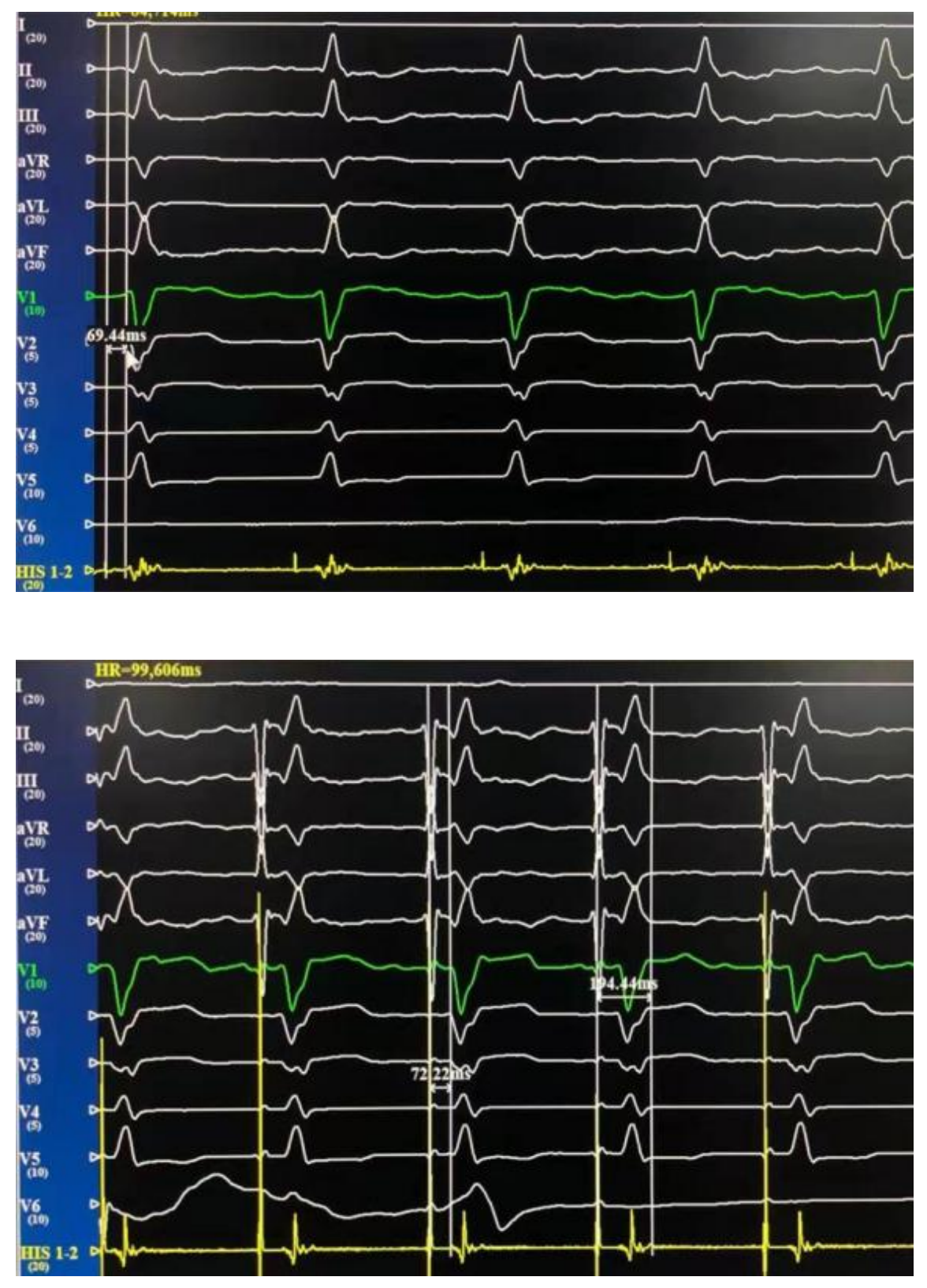

His potential His lead pacing 
LBP 
RAO LAO

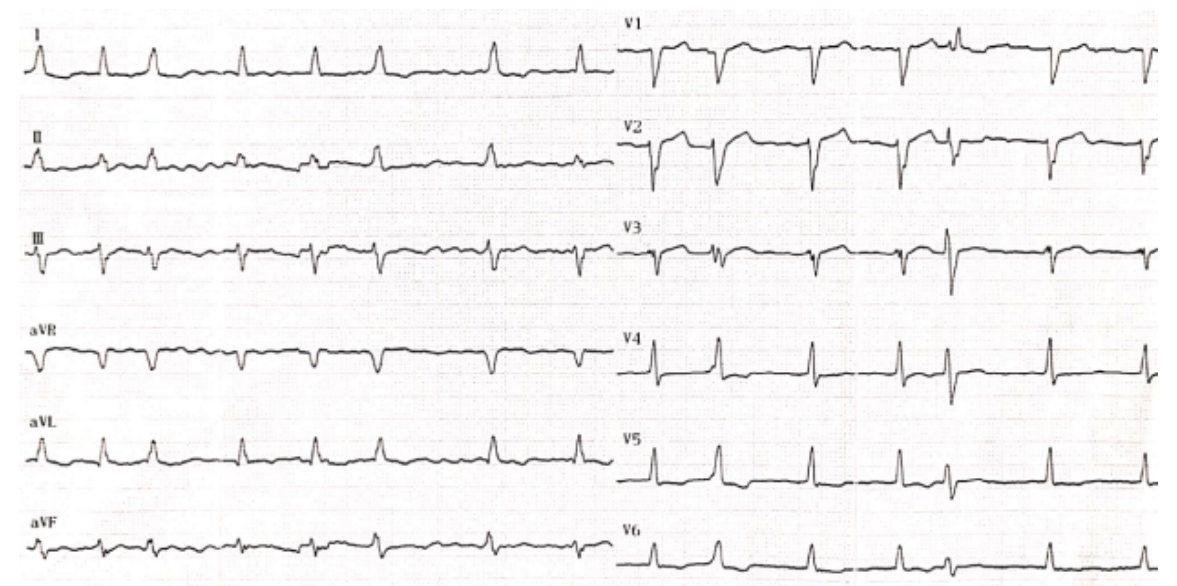



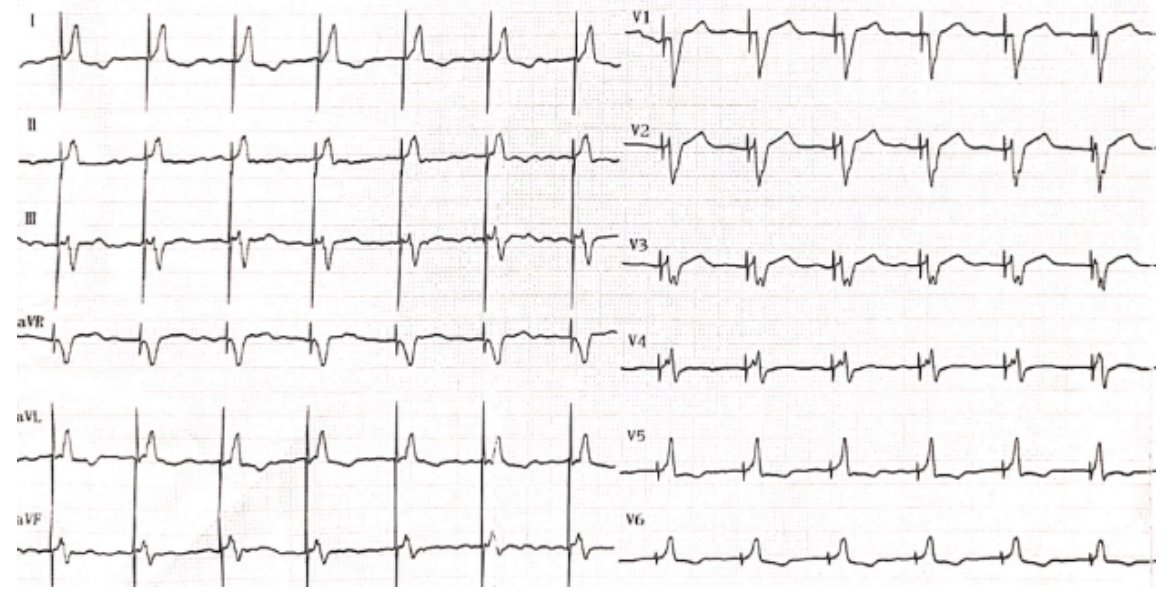

Native ECG His bundle pacing

Left bundle-branch pacing: Select Secure lead (model 3830, Medtronic Inc) was delivered through a delivery sheath (model C315His, Medtronic Inc). During the lead placement procedure, the 12-lead ECG and intracardiac electrogram via pacing lead were monitored and recorded. Under fluoroscopic imaging in the $30^{\circ}$ right anterior oblique view, His bundle potential was first identified and the His bundle region was used as an anatomical marker. Then the sheath and the pacing lead were moved by $1.0-1.5 \mathrm{~cm}$ in the cardiac apex direction and the pacing lead was perpendicularly screwed into IVS until the pacing lead helix to the left side of the IVS. LBP presented an ECG QRS pattern of right bundle branch block, with reduced time interval between stimulation and peak left ventricular activation in ECG leads V5 and V6. The lower rate for HBP was initially set at 70 beats/min.

Left bundle branch pacing criteria: (1) The morphological manifestations of rhythmic QRS are right bundle branch block; (2) Left bundle branch potential can be identified with PV interval of 20-30ms, but the left bundle branch potential prior to $\mathrm{V}$ wave can not be identified during left bundle branch block; (3) Peak left ventricular activation time (LVAT) is shortened, usually less than 80ms (lead V5 or V6 ). 


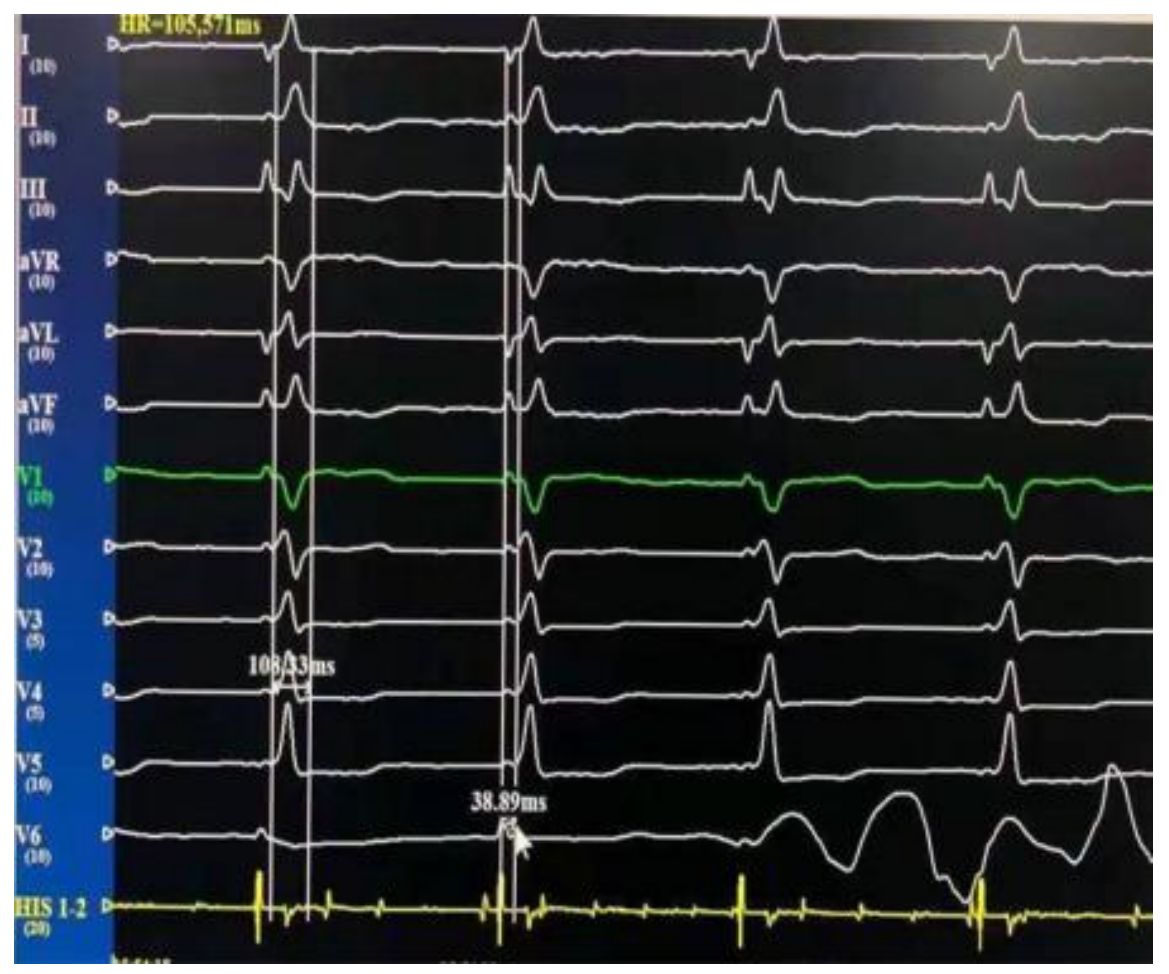



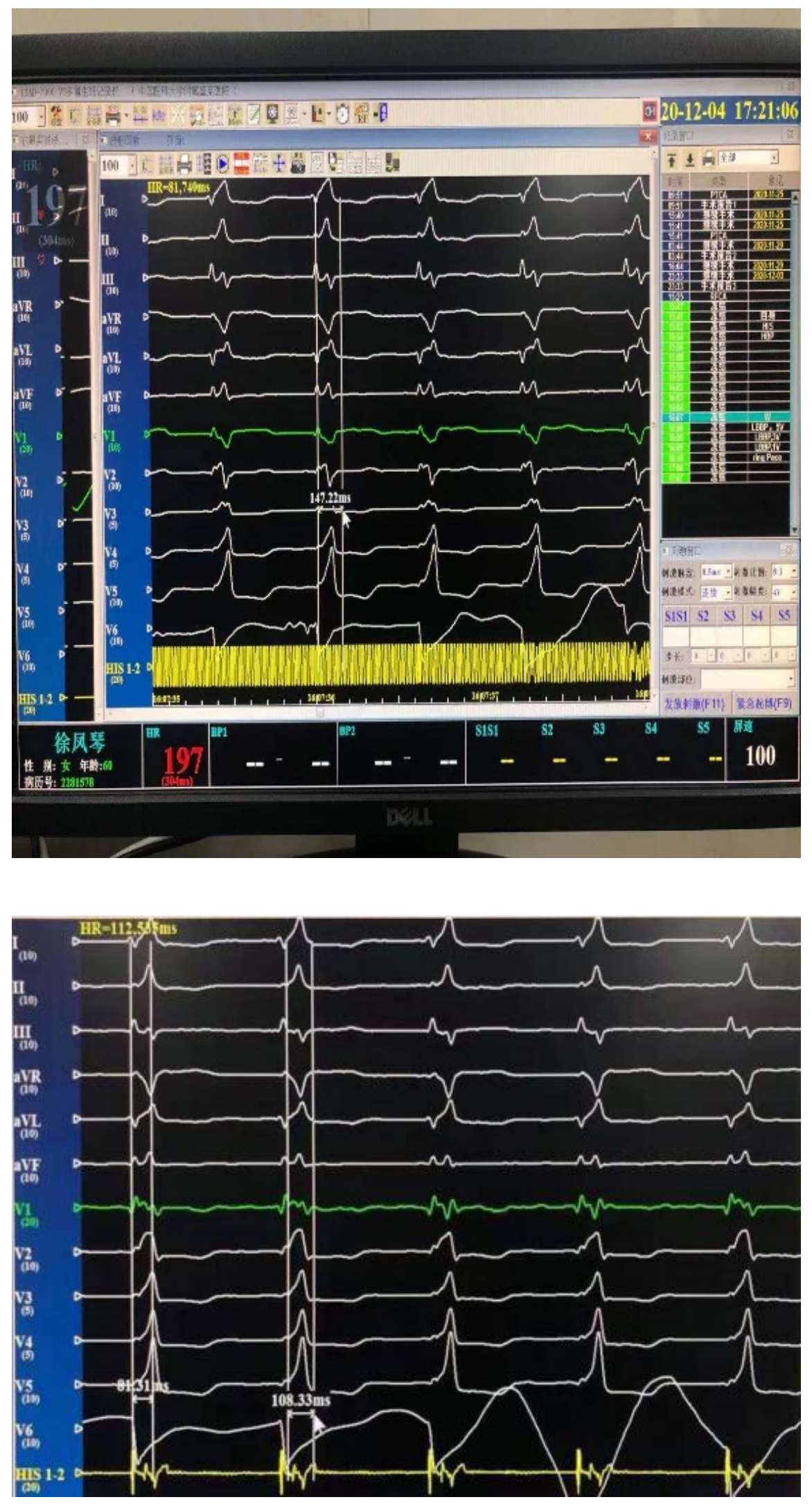


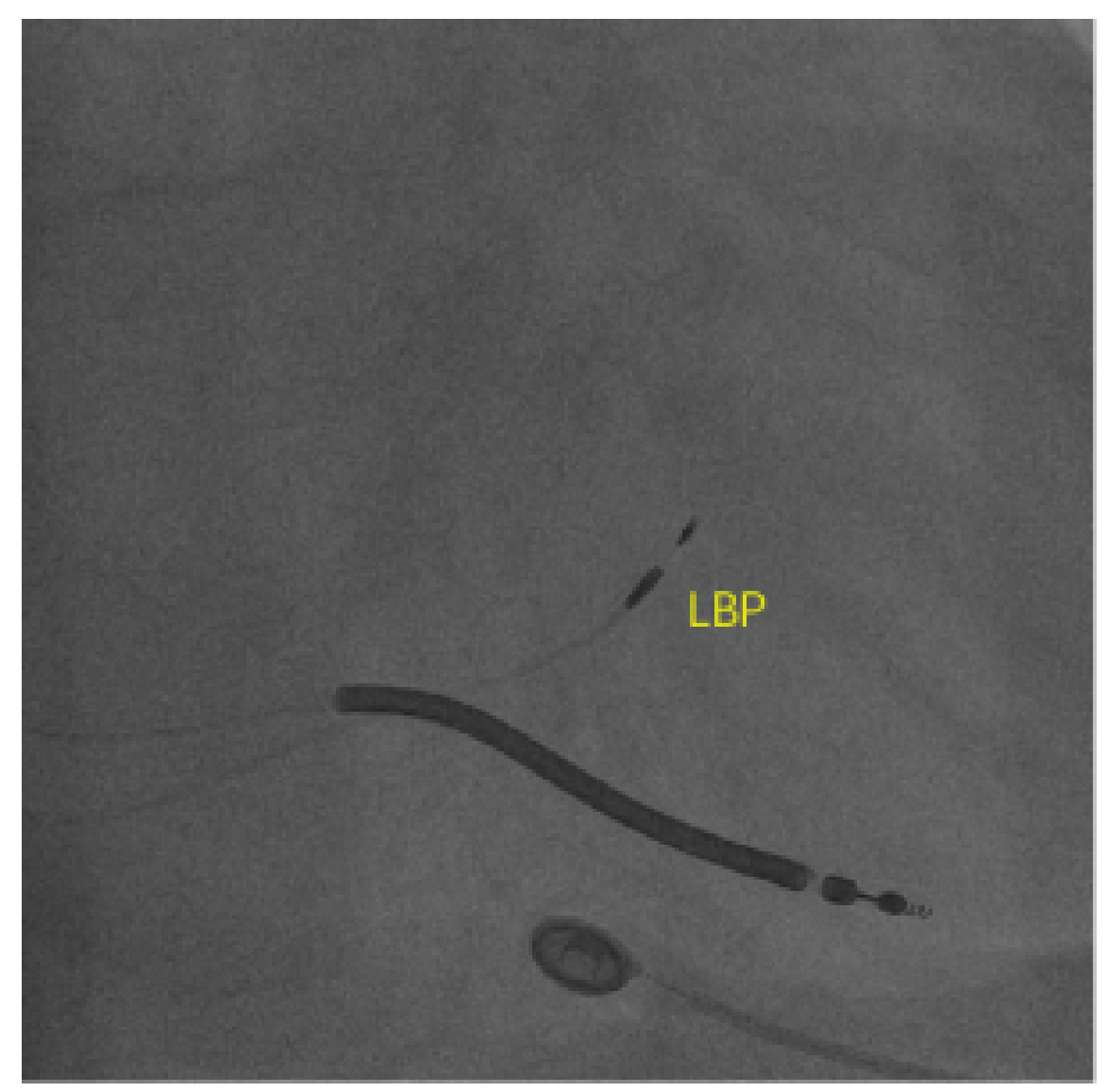




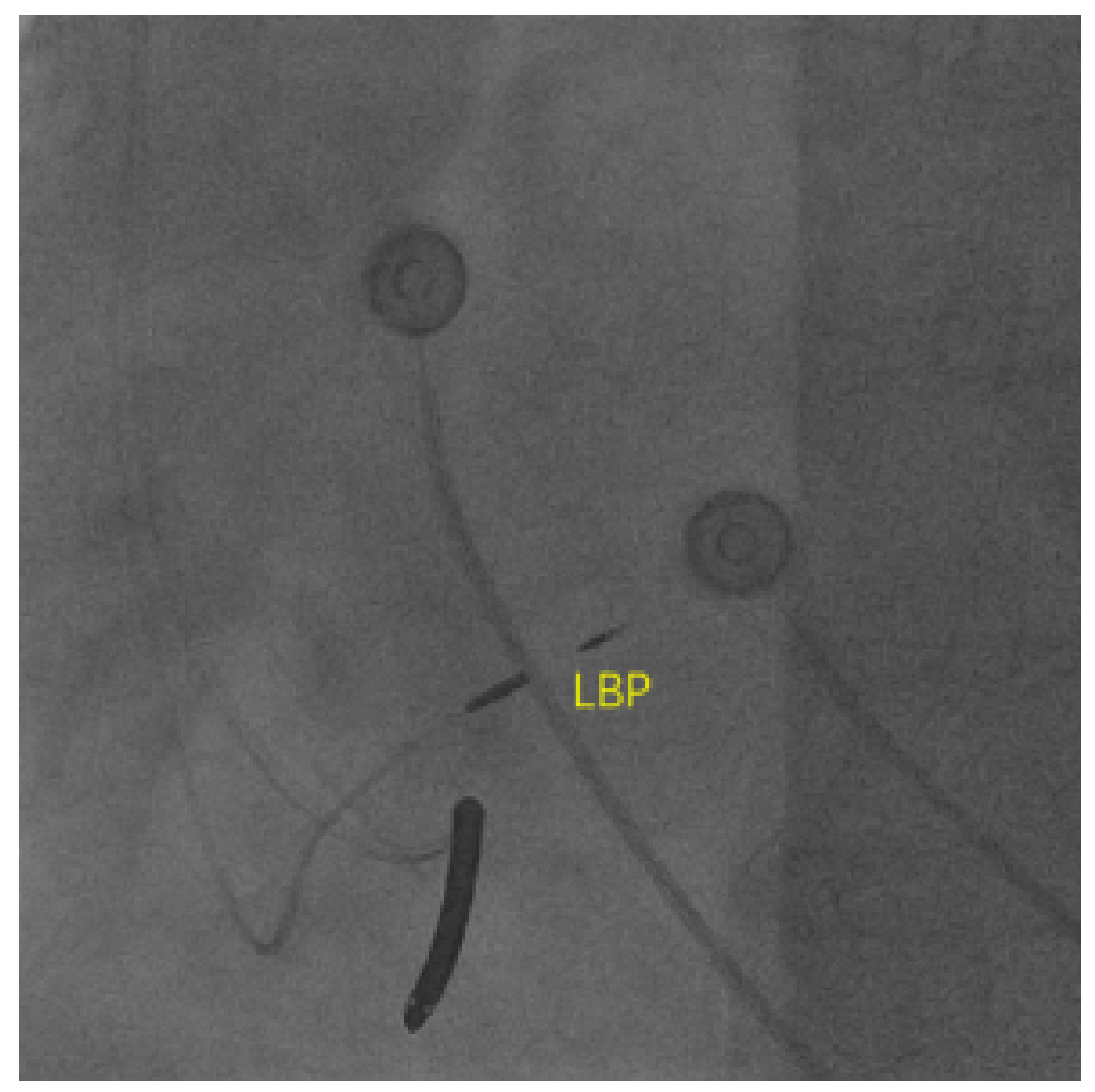

Native ECG Target Peak LVAT RAO LAO

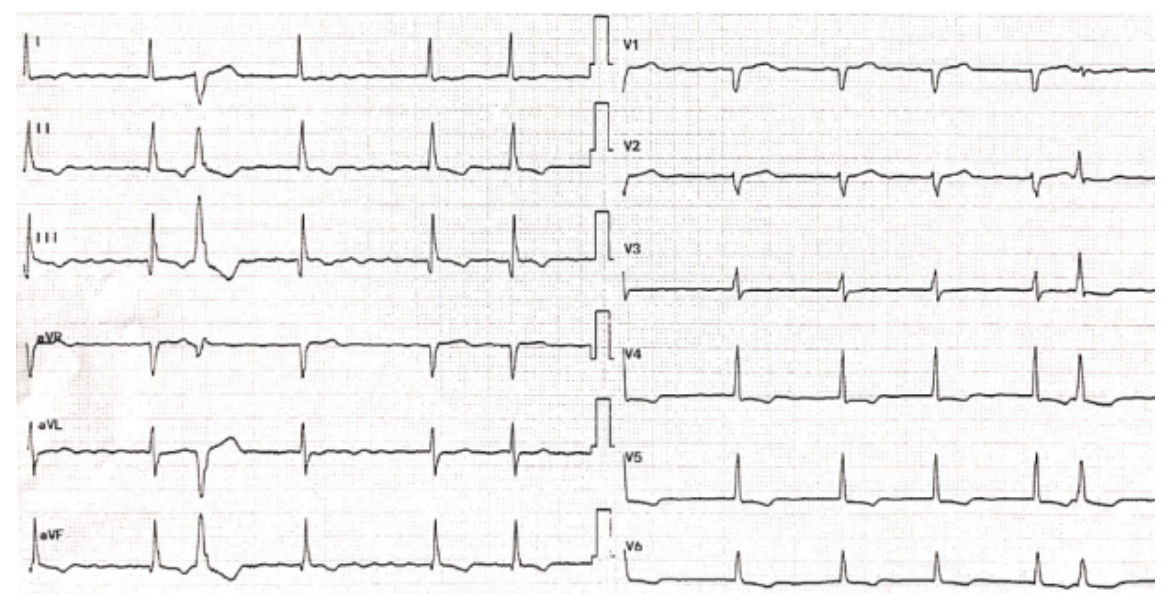




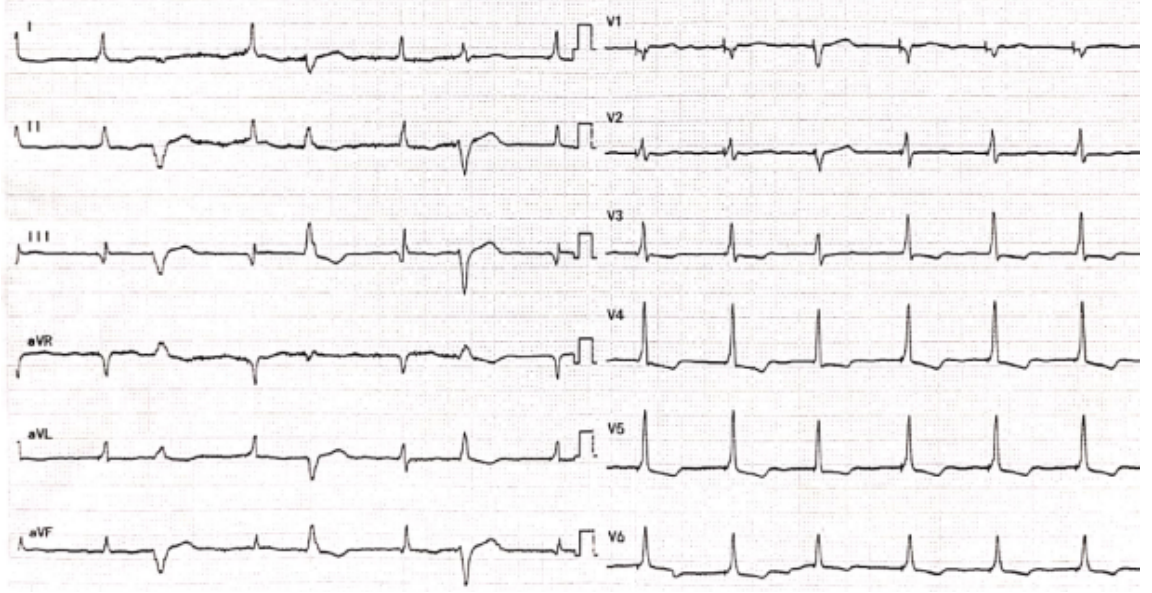

Native ECG Left bundle-branch pacing

\section{Follow Up}

Patients were followed in clinic at 1 month, 3 months, 6 months. Pacing thresholds, sensed R-wave amplitudes, lead impedance and percentages of ventricular pacing were recorded at each visit. Routine ECG examination, NT-proBNP (N-terminal pro-brain natriuretic peptide) test were performed, and echocardiographic indices including left ventricular end diastolic dimension (LVEDD) and left ventricular ejection fraction (LVEF) were measured during follow-up. At each follow-up visit, the dosage of $\beta$-blocker was adjusted according to pacing proportion, and the ventricular rate was controlled less than $60-80$ beats / min as far as possible. The pacing rate was programmed to $60-80$ beats / min. If necessary, the pacing rate set by the program could be increased according to the higher pacing ratio. After the pacemaker was implanted, patients who were readmitted to the hospital due to an event of heart failure and cardiac death would be seen by phone, and the date of the event would be recorded.

\section{Statistical Analyses}

Continuous variables were expressed as mean \pm standard deviation (SD) in normal distribution and median \pm quartile in non-normal distribution. Categorical variables were presented as number of patients (\%).Receiver operator characteristic (ROC) analysis was performed to determine the optimal cut-off point of pacing proportion to predict MACE and the area under curve (AUC) was calculated as a measure of test accuracy. Paired $\mathrm{T}$ tests were performed to compare the differences between the baseline time point and half year follow-up time point. The independent sample $\mathrm{T}$ test was used for normal distribution continuous variables to compare the baseline characteristics between high and low pacing proportion, Mann Whitney $\mathrm{U}$ test was used for non-normal distribution continuous variables, and Pearson Chi-square test was used for category variables. MACE (major adverse cardiovascular events) rate curves were constructed using the KaplanMeier method stratified by high and low pacing proportion and were compared by log rank tests. All data management and statistical analyses were carried out using the SPSS version 24.0. All statistical tests were two-tailed, and $\mathrm{P}<0.05$ was considered to be statistically significant.

Figure 1. Schematic summary of study and patient flow

\section{Results}

\section{Implantation Results, Device Electrical Parameters and Patient Characteristics}

In all 37 enrolled patients, His-Purkinje system pacing were attempted (Figure 1). Failure of HPSP occurred in 5 of these patients (13.5\%). HPSP was achieved in the remaining 32 patients (86.5\%). 2 patients did not achieve permanent HPSP due to thresholds rising (5.4\%). Of the 30 patients with HPSP, 17 patients 
attempted permanent HBP (6 with S-HBP and 11 with NS-HBP), and 13 patients attempted LBP. HisPurkinje system pacing was performed in 13 patients with single-chamber pacemakers, 11 patients with dual-chamber pacemakers, 5 patients with dual-chamber implanted defibrillators, and 1 patient with CRT pacemakers.

HBP threshold was $1.29 \pm 0.47 \mathrm{~V}$ and $1.52 \pm 0.82 \mathrm{~V}$ at baseline and half year follow-up, respectively. LBP threshold was $0.88 \pm 0.22 \mathrm{~V}$ and $0.78 \pm 0.28 \mathrm{~V}$ at baseline and half year follow-up, respectively. HBP lead impedance did not significantly change at half year follow-up (from $357.5 \pm 25.6 \Omega$ at baseline to $362.1 \pm 45.7 \Omega$ at half year follow-up). LBP lead impedance changed from $608.3 \pm 69.9 \Omega$ at baseline to $574.3 \pm 77.2 \Omega$ at half year follow-up.

Left bundle branch block (LBBB) was present in 10 patients. Right bundle branch block (RBBB) was present in 5 patients. The native QRS duration (QRSd) of 30 patients was $121.4 \pm 29.5 \mathrm{~ms}$, and the pacing QRSd was shortened to $111.8 \pm 15.9 \mathrm{~ms}$.

The follow-up period was $15.1 \pm 9.4$ months. The median follow-up period was 12.0 months. During the followup period, 2 patients were readmitted to hospital due to heart failure and 4 patients died of cardiac origin. MACE was defined as the composite endpoint of readmission for heart failure and cardiac mortality. Figure 2 shows the predictive ability of pacing proportion for MACE by ROC analysis. The AUC of pacing proportion for predicting MACE was 0.830 ( $\mathrm{SE}=0.140,95 \% \mathrm{CI}: 0.649-0.941, \mathrm{P}=0.018$ ), indicating that pacing proportion had a significant predictive value for the prognosis of atrial fibrillation and heart failure patients. ROC analysis showed that the optimal threshold for pacing ratio to predict MACE was 71\%(sensitivity:83.3\%, specificity: $91.7 \%)$.

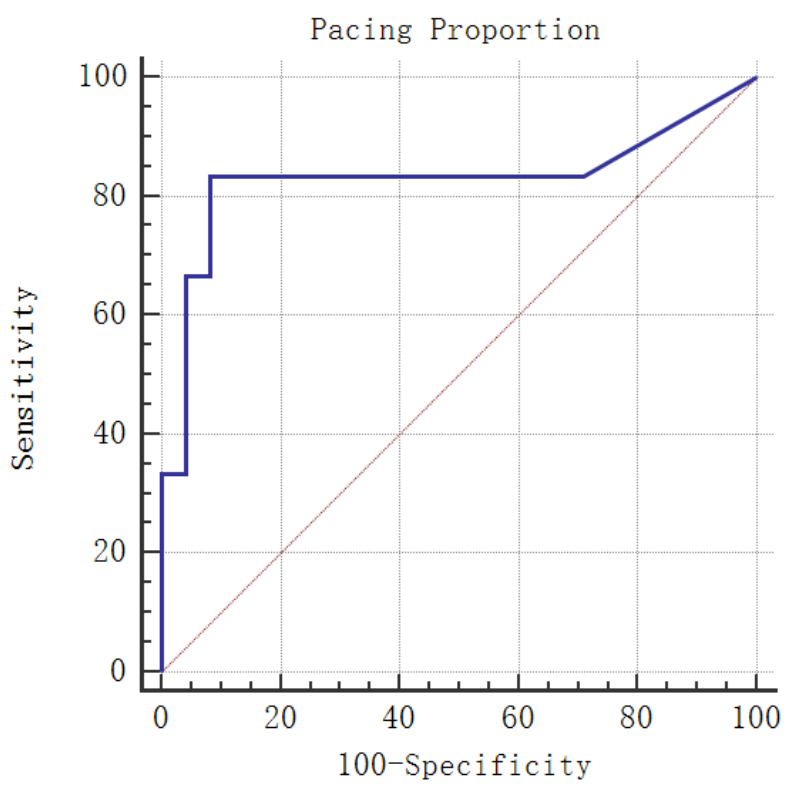

Figure 2. ROC analysis of pacing proportion for prediction of MACE

\section{Patient Baseline Characteristics under High and Low Pacing Proportion}

Pacing proportion $>71 \%$ was defined as the high pacing proportion group ( $\mathrm{N}=23$ ), whereas pacing proportion[?]71\% was defined as the low pacing proportion group $(\mathrm{N}=7)$. Detailed baseline characteristics of patients under high and low pacing proportion were summarized in Table 1. Characteristics of patients such as gender, age, systolic blood pressure, heart rate, hypertension, diabetes, coronary heart disease, PCI 
history, smoking history, hemoglobin, albumin, creatinine, NT-proBNP, native QRS duration, LVEF, HBP were comparable between high and low pacing proportion group (all $\mathrm{P}>0.05$ ). The dosage of $\beta$-blockers in high pacing proportion was significantly lower than that in low pacing proportion $(\mathrm{P}=0.018)$.

Table 1. Comparison of Patient Baseline Characteristics with High and Low Pacing Proportion

\begin{tabular}{llll}
\hline Variables & High pacing proportion(n=23) & Low pacing proportion(n=7) & $P$ value \\
\hline Epidemiologics & Epidemiologics & Epidemiologics & Epidemi \\
Gender (males: n, \%) & $15(65.2 \%)$ & $5(71.4 \%)$ & 0.760 \\
Age (years) & $74.0 \pm 10.8$ & $69.0 \pm 6.6$ & 0.157 \\
Admission vital signs & Admission vital signs & Admission vital signs & Admissic \\
Admission systolic blood pressure (mmHg) & $141.7 \pm 26.5$ & $131.3 \pm 26.9$ & 0.373 \\
Admission heart rate (beats/min) & $84.5 \pm 25.2$ & $89.4 \pm 36.3$ & 0.685 \\
Co-morbidities & Co-morbidities & Co-morbidities & Co-morb \\
Hypertension (n, \%) & $14(60.9 \%)$ & $5(71.4 \%)$ & 0.612 \\
Diabetes mellitus (n, \%) & $9(39.1 \%)$ & $3(42.9 \%)$ & 0.860 \\
Coronary heart disease (n, \%) & $11(47.8 \%)$ & $3(42.9 \%)$ & 0.818 \\
PCI history (n, \%) & $2(8.7 \%)$ & $1(14.3 \%)$ & 0.666 \\
Smoking history (n, \%) & $9(39.1 \%)$ & $4(57.1 \%)$ & 0.400 \\
Lab examination & Lab examination & Lab examination & Lab exar \\
Hemoglobin (g / L) & $134.3 \pm 22.6$ & $146.7 \pm 24.8$ & 0.222 \\
Albumin (g / L) & $36.9 \pm 4.4$ & $35.7 \pm 3.6$ & 0.531 \\
Creatinine (umol / L) & $91.4 \pm 22.1$ & $92.6 \pm 20.1$ & 0.897 \\
NT-proBNP(ng/L) & $1740(1108-4123)$ & $3013(1406-4908)$ & 0.207 \\
Native QRSd (ms) & $120.0 \pm 30.2$ & $125.7 \pm 29.1$ & 0.664 \\
LVEF (\%) & $46.4 \pm 14.6$ & $42.3 \pm 16.3$ & 0.525 \\
Drug and device therapy & Drug and device therapy & Drug and device therapy & Drug an \\
HBP (n, \%) & $13(56.5 \%)$ & $4(57.1 \%)$ & 0.977 \\
Pacing proportion(\%) & $93.7 \pm 8.6$ & $54.1 \pm 16.8$ & $i 0.001$ \\
$\beta$-blockers (mg daily) & $59.9 \pm 52.1$ & $120.6 \pm 68.5$ & 0.018 \\
Angiotensin-converting enzyme inhibitor (n, \%) & $15(65.2 \%)$ & $7(100.0 \%)$ & 0.068 \\
Diuretics (n, \%) & $7(30.4 \%)$ & $4(57.1 \%)$ & 0.199 \\
\hline & & & \\
\hline
\end{tabular}

Mean $\pm \mathrm{SD}$ or $\mathrm{n}(\%)$ unless specified; * Median and interquartile range

\section{Clinical Outcomes and Echocardiographic Changes of Patients under High and Low Pacing Proportion}

After half year of HPSP, there were significant overall improvements in NYHA classification, NT-proBNP concentration and LVEF in high pacing proportion group at half-year follow-up from the baseline. There were no significant changes of NT-proBNP concentration, LVEF and LVEDD in low pacing proportion group at half-year follow-up from the baseline(Table 2).

Table 2. Comparison of Clinical Outcomes and Echocardiographic Changes of Patients under High and Low Pacing Proportion - Baseline vs Half- year Follow-up

\begin{tabular}{llllll}
\hline & & NYHA class & NT-proBNP(ng/L) & LVEDD $(\mathrm{mm})$ & LVEF $(\%)$ \\
\hline High Pacing proportion(n=23) & Baseline & $3.1 \pm 0.8$ & $2916 \pm 2849$ & $55.1 \pm 8.3$ & $46.0 \pm 14.7$ \\
& Half year follow-up & $1.6 \pm 0.7$ & $1187 \pm 1609$ & $52.4 \pm 7.1$ & $53.4 \pm 10.5$ \\
& $P$ value & $i 0.001$ & 0.014 & 0.009 & 0.001 \\
Low Pacing proportion(n=7) & Baseline & $3.4 \pm 0.5$ & $3720 \pm 2913$ & $63.0 \pm 13.7$ & $44.1 \pm 16.0$ \\
& Half year follow-up & $2.5 \pm 1.1$ & $2428 \pm 2035$ & $62.1 \pm 12.9$ & $44.9 \pm 13.7$
\end{tabular}




\begin{tabular}{lllll}
\hline & NYHA class & NT-proBNP $(\mathrm{ng} / \mathrm{L})$ & LVEDD $(\mathrm{mm})$ & $\operatorname{LVEF}(\%)$ \\
\hline$P$ value & 0.021 & 0.206 & 0.429 & 0.700 \\
\hline
\end{tabular}

\section{Clinical Outcomes and Echocardiographic Changes of Patients with Different QRS Duration and LVEF under High and Low Pacing Proportion}

All patients were divided into two subgroups based on QRS duration: wide QRS group with QRS $>120 \mathrm{~ms}$ $(\mathrm{N}=15)$ and narrow QRS group with QRS[?]120ms $(\mathrm{N}=15)$; and they were also divided into two subgroups based on LVEF: the HFpEF group with LVEF [?]40\% ( $=18)$ and HFrEF group with LVEF<40\% (N=12).

In condition of high pacing proportion $(>71 \%)$, NT-proBNP concentration was reduced to $1085 \pm 2074 \mathrm{ng} / \mathrm{L}$ after half year of HPSP from the baseline $2757 \pm 2835 \mathrm{ng} / \mathrm{L}$ in patients of QRSd $>120 \mathrm{~ms}(\mathrm{P}=0.010)$, and to $1219 \pm 1032 \mathrm{ng} / \mathrm{L}$ from baseline $2930 \pm 2897 \mathrm{ng} / \mathrm{L}$ in the patients of $\mathrm{QRSd}[?] 120 \mathrm{~ms}(\mathrm{P}=0.032)$. NYHA classification was improved to 1.6+-0.9 after half year of HPSP from the baseline 3.2+-0.8 in patients of QRSd $>120 \mathrm{~ms}(\mathrm{P}<0.001)$, and to 1.6+-0.5 after HPSP from the baseline 3.0+-0.7 in patients of QRSd[?]120ms $(\mathrm{P}<0.001)$. NT-proBNP concentration was reduced to $1744+-2472 \mathrm{ng} / \mathrm{L}$ after half year of HPSP from the baseline $4205+-4044 \mathrm{ng} / \mathrm{L}$ in $\mathrm{HFrEF}$ patients $(\mathrm{P}=0.032)$, and to $840+-747 \mathrm{ng} / \mathrm{L}$ from baseline $2123+-1598$ $\mathrm{ng} / \mathrm{L}$ in the HFpEF patients $(\mathrm{P}=0.010)$. NYHA classification was improved to $1.9+-1.0$ after half year of HPSP from the baseline 3.5+-0.8 in HFrEF patients $(\mathrm{P}=0.003)$ and to $1.4+-0.5$ after HPSP from the baseline $2.8+-0.6$ in HFpEF patients $(\mathrm{P}<0.001)$.

In condition of low pacing proportion([?]71\%), after half year of HPSP, there were no significant changes of NT-proBNP concentration and NYHA classification in patients of QRSd $>120 \mathrm{~ms}(2259+-2107 \mathrm{ng} / \mathrm{L}$, versus baseline $2760+-1634 \mathrm{ng} / \mathrm{L}, \mathrm{P}=0.529 ; 2.8+-1.1$, versus baseline $3.6+-0.5, \mathrm{P}=0.099)$, and QRSd[?]120ms(2709+$2331 \mathrm{ng} / \mathrm{L}$, versus baseline $5320+-4267 \mathrm{ng} / \mathrm{L}, \mathrm{P}=0.359 ; 2.0+-0.0$, versus baseline $3.0+-0.0, \mathrm{P}=0.225)$. After half year of HPSP, there were no significant changes of NT-proBNP concentration and NYHA classification in $\mathrm{HFrEF}$ patients $(2870+-2107 \mathrm{ng} / \mathrm{L}$, versus baseline $5111+-3399 \mathrm{ng} / \mathrm{L}, \mathrm{P}=0.306 ; 3.0+-0.8$, versus baseline $3.8+-0.5, \mathrm{P}=0.058$ )and HFpEF patients $(1985+-2174 \mathrm{ng} / \mathrm{L}$, versus baseline $2329+-1756 \mathrm{ng} / \mathrm{L}, \mathrm{P}=0.363 ; 2.0+-$ 1.2 , versus baseline $3.0+-0.0, \mathrm{P}=0.182$ ).

Echocardiographic changes of patients with different QRS duration and LVEF under high and low pacing proportion were summarized in Figure 3. Compared with baseline echocardiographic parameters, LVEF significantly increased while LVEDD decreased in HFrEF and wide QRS (QRSd>120ms) patients when pacing proportion was more than $71 \%$. However, moderate but no significant improvements of LVEF and LVEDD were observed in HFpEF and narrow QRS (QRSd[?]120ms) patients. In condition of low pacing proportion([?]71\%), HFrEF and HFpEF patients showed no significant change in LVEF and LVEDD after half year of HPSP treatment regardless of QRS duration.
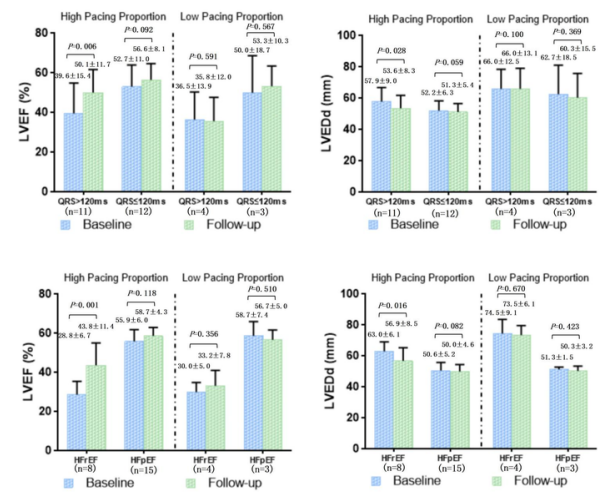
Figure3. Comparison of Echocardiographic Changes of Patients with different QRS Duration and LVEF under High and Low Pacing Proportion - Baseline vs Half-year Follow-up

Figure 4 shows the Kaplan-Meier curves MACE rate stratified by pacing proportion level. It was found that patients in low pacing proportion group had significantly higher MACE rate than patients in high pacing proportion $(\mathrm{P}<0.001)$.

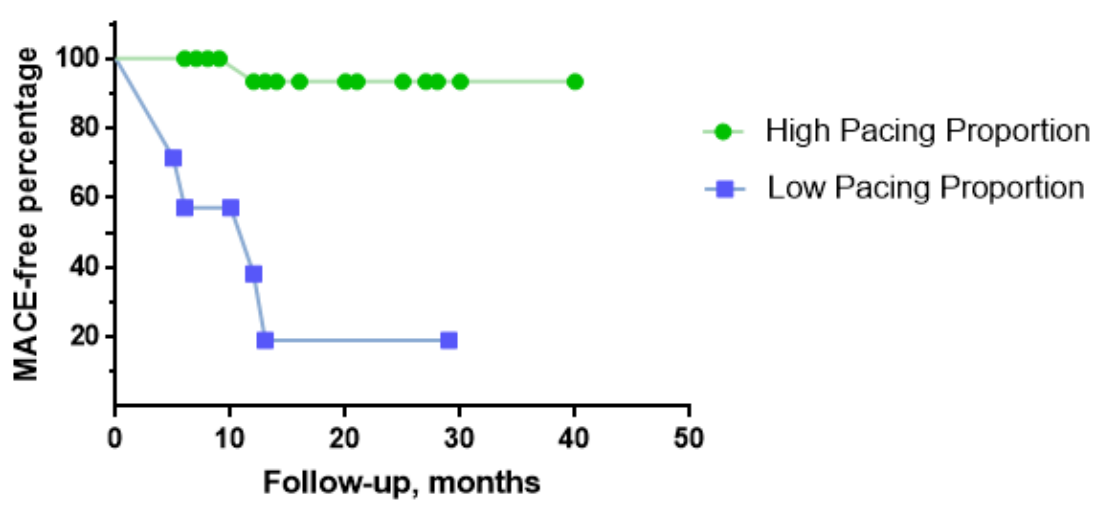

Figure 4. Kaplan-Meier estimate for MACE by high and low pacing proportion

\section{Discussion}

Ventricular rhythm is irregular in AF with or without rapid ventricular rate. Fast ventricular rate has an acknowledged deleterious impact upon left ventricular (LV) systolic function ${ }^{12,13}$, and ventricular irregular rhythm itself also has adverse effects on LV systolic function ${ }^{4}$. Therefore, the treatment of AF should focus on rate control and rhythm control. HBP combined with AVN ablation can not only achieve rate control and rhythm control, but also utilize the complete His-Purkinje pathway, which is beneficial to synchronous ventricular activation ${ }^{14}$. If $\mathrm{AF}$ is complicated with bundle branch block and wide QRS complex, whether LBBB or RBBB pattern, HPSP can overcome ventricular systolic asynchrony and improve cardiac function by correcting conduction block ${ }^{15,16}$. In patients with atrial fibrillation without AVN ablation, $\beta$-blockers can inhibit AVN conduction function and reduce intrinsic heart rate to achieve high proportion of HPSP and fulfill the purpose of rate control and rhythm control.

In this study, we found that high proportion of His-Purkinje system pacing could significantly improve the clinical outcomes and echocardiographic results of atrial fibrillation patients complicated with heart failure. However, the clinical characteristics such as age, gender, past history, baseline of liver and kidney function and cardiac function in high pacing proportion patients were similar to those in low pacing proportion. Therefore, the discrepancy of therapeutic effect between high and low pacing proportion group could be attributed to high His-Purkinje system pacing proportion itself. Previous studies demonstrated that increasing the dose of $\beta$-blockers was an independent factor to improve the prognosis of $\mathrm{HF}$ patients treated with $\mathrm{CRT}^{17}$. In the present study, $\beta$-blockers dosage $(59.9 \pm 52.1 \mathrm{mg}$ daily) in high pacing proportion group was significantly lower than that $(120.6 \pm 68.5 \mathrm{mg}$ daily $)$ in low pacing proportion group $(\mathrm{P}=0.018)$, indicating that the clinical benefits of patients in high pacing proportion group were further ascribed to the higher pacing proportion.

Previous studies have showed that improvements in heart failure symptoms using NYHA classification based on severity, reduction of LVEDD, improvement of LVEF, were observed cardiac resynchronization therapy (CRT) in wide QRS $(159.2 \pm 28.6 \mathrm{~ms})$ patients with atrial fibrillation and heart failure by implanting His bundle lead ${ }^{15}$. In this study, HBP achieved an average pacing percentage of $97 \%$ through the optimization of medical therapy and appropriate device programming ${ }^{15}$. Previous studies demonstrated that AF was associated with poor prognosis in patients with CRT. Atrial fibrillation leads to a decrease in the proportion 
of biventricular pacing (BivP). BivP[?]98\% is an independent risk factor for poor prognosis in patients with $\mathrm{CRT}^{18}$. The high pacing proportion of BivP was defined as $>98 \%$. When BivP $>98 \%$, it could significantly reduce mortality rate ${ }^{19}$. The present study found that the effect could be observed when the proportion of HPSP was more than $71 \%$ in patients with wide QRS AF and HF. The discrepancy might be caused by the difference of native QRSd and pacing modes in study population. QRS duration of the wide QRS patients in the present study was $146.1+-18.9 \mathrm{~ms}$, thus the degree of ventricular activation asynchrony was lower than that of the patients with previous CRT implantation. The detrimental influence of native activation on ventricular remodeling was relatively small, suggesting a relatively low pacing proportion needed to achieve clinical benefits. Furthermore, the physiology of BivP was inferior to that of HPSP. Some studies found that HBP could provide better ventricular resynchronization and hemodynamic feedback than $\mathrm{BiVP}^{20}$. On the basis of the above reasons, it is preliminarily explained why the pacing proportion is relatively low enough to improve the clinical condition. Previous studies on paroxysmal AF complicated with HF revealed that the longer the sinus rhythm time ([?]61\%) was maintained, the more significant the improvement of life quality, 6-minute walk test and NYHA classification were observed ${ }^{21}$. For persistent AF patients with HPSP, ventricular activation sequence and rhythm are similar to those of sinus rhythm. However, considering atrial fibrillation has not been corrected, the atrium loses contraction function and impairs $20 \%$ of cardiac output compared with sinus rhythm in patients with paroxysmal $\mathrm{AF}^{22}$. Therefore, pacing proportion greater than $61 \%$ ( $71 \%$ in this study) was required to achieve the purpose of treatment. Furthermore, QRS duration of the patients included in this study was $114+-30 \mathrm{~ms}^{21}$, ventricular synchronization was better than that of the present study (QRSd=120.0+-30.2 ms). As for HPSP application for patients with persistent AF and $\mathrm{HF}$, the analysis above gives a tendency that when the native QRS duration is greater than pacing QRS duration, the longer the native QRS duration is, the higher pacing proportion of resynchronization therapy is needed to achieve clinical benefits. There could be a lower limit for the pacing proportion required for the effective treatment, but the establishment of the lower limit still needs further exploration.

In patients with narrow QRS $(<120 \mathrm{~ms})$ persistent AF and HF, regular paced ventricular rhythm by HPSP was a primary hemodynamic benefit due to the absence of bundle branch block ${ }^{23}$. The present study found that there were significant reductions of NYHA classification and NT-proBNP concentration when high proportion pacing was applied, but no significant improvements were observed in echocardiographic measurements. Deshmukh et al. performed AVN ablation and HBP in patients with narrow QRS $(<120 \mathrm{~ms})$ $\mathrm{AF}$ and dilated cardiomyopathy, which showed the improvement of LVEDD and $\mathrm{LVEF}^{9}$. Huang et al. implemented AVN ablation and HBP for patients with narrow QRS complex (107.1+-25.8ms) AF and HF, and the echocardiographic parameters were also improved ${ }^{10}$. Compared with the results of previous studies, the difference in the improvement of echocardiography in the present study was related to the fact that our subjects did not undergo AVN ablation, and the pacing proportion was less than $100 \%$. Therefore, AVN ablation was recommended for these patients to increase the pacing proportion to $100 \%$, in order to further improve the therapeutic effect.

The present study found that His-Purkinje system pacing proportion had a good predictive ability for MACE in patients with persistent atrial fibrillation and heart failure $(\mathrm{AUC}=0.830)$. The optimal cut-off point of pacing proportion for predicting MACE related to prognosis was $71 \%$ during the follow-up period of 15.1 +- 9.4 months. When patients were divided into groups by pacing proportion of $71 \%$, patients with QRS > $120 \mathrm{~ms}$ and HFrEF in high proportion pacing group could show significant improvement in clinical outcomes and echocardiographic results within 6 months after HPSP, which were similar to the results in the study of Huang et $\mathrm{al}^{10}$. However echocardiographic parameters LVEF increased from baseline $52.7+-11.0 \%$ to 56.6 +- 8.1\%, LVEDD decreased from baseline 52.2 +- $6.3 \mathrm{~mm}$ to $51.3+-5.4 \mathrm{~mm}$ in patients with QRS [?] $120 \mathrm{~ms}$, LVEF increased from baseline $55.9+-6.0 \%$ to $58.7+-4.3 \%$, LVEDD decreased from baseline 50.6 +$5.2 \mathrm{~mm}$ to 50.0 +- $4.6 \mathrm{~mm}$ in HFpEF patients. Unlike previous studies, the improvement was modest, but not significant ${ }^{10}$. The discrepancy perhaps resulted from the absence of AVN ablation and pacing proportion being less than $100 \%$. However, considering that the MACE prognosis in the high pacing ratio group was better than that in the low pacing ratio group, this difference was likely to be explained by the fact that the 6-month follow-up time was significantly shorter than the average follow-up time of 21.1+-9.3 months in the 
study of Huang et $\mathrm{al}^{10}$. Therefore, it is suggested that high His-Purkinje system pacing proportion through the usage of $\beta$-blockers had an therapeutic efficacy for patients with atrial fibrillation and heart failure.

\section{Study limitations}

This was a retrospective, observational single center study with a small patient population. We expected to perform a large-scale multicenter prospective clinical trial in the future. Furthermore, this study belonged to the self-control study and lacked a control group, so the differences in therapeutic effects of the His-Purkinje pacemaker treatment group, internal medicine treatment group and catheter ablation group could not be obtained. Randomized controlled trials are expected to be conducted in the future to compare the differences in therapeutic effects of each treatment method. In this study, patients with pacing proportion greater than $71 \%$ achieved significant clinical benefits. However, due to the small size of the study population and unevenly distributed pacing proportion, the high pacing proportion amounting to $71 \%$ could only indicate that the higher the pacing proportion, the greater the clinical benefit. And it could not be interpreted as the lower limit of pacing ratio to achieve effective therapeutic effect. Large-scale observations are necessary to establish a lower limit for pacing proportion.

\section{Reference}

1 Anter E, Jessup M, Callans DJ. Atrial fibrillation and heart failure: treatment considerations for a dual epidemic. Circulation. 2009;119(18):2516-25.

2 Kirchhof P, Benussi S, Kotecha D, et al. 2016 ESC guidelines for the Management of Atrial Fibrillation Developed in collaboration with EACTS. Rev Esp Cardiol (Engl Ed). 2017;70(1):50.

3 Rosenberg MA, Manning WJ. Diastolic dysfunction and risk of atrial fibrillation: a mechanistic appraisal. Circulation.2012;126(19):2353-62.

4 Prabhu S, Voskoboinik A, Kaye DM, et al. Atrial Fibrillation and Heart Failure - Cause or Effect? Heart Lung Circ. 2017;26(9):967-974.

5 Wang S, Wu S, Xu L, et al. Feasibility and Efficacy of His Bundle Pacing or Left Bundle Pacing Combined With Atrioventricular Node Ablation in Patients With Persistent Atrial Fibrillation and Implantable Cardioverter-Defibrillator Therapy. J Am Heart Assoc. 2019;8(24):e014253.

6 Sultan A, Lüker J, Andresen D, et al. Predictors of Atrial Fibrillation Recurrence after Catheter Ablation: Data from the German Ablation Registry. Sci Rep.2017;7(1):16678.

7 Verma A, Jiang CY, Betts TR, et al. Approaches to catheter ablation for persistent atrial fibrillation. N Engl J Med. 2015;372:1812-22.

8 Pallisgaard JL, Gislason GH, Hansen J, et al. Temporal trends in atrial fibrillation recurrence rates after ablation between 2005 and 2014: a nationwide Danish cohort study. Eur Heart J. 2018;39(6):442-449.

9 Deshmukh P, Casavant DA, Romanyshyn M, et al. Direct His-bundle pacing: a novel approach to cardiac pacing in patients with normal His-Purkinje activation. Circulation. 2000;101(8):869-77.

10 Huang W, Su L, Wu S, et al. Benefits of Permanent His Bundle Pacing Combined With Atrioventricular Node Ablation in Atrial Fibrillation Patients With Heart Failure With Both Preserved and Reduced Left Ventricular Ejection Fraction. J Am Heart Assoc. 2017 ;6(4):e005309.

11 Vijayaraman P, Subzposh FA, Naperkowski A. Atrioventricular node ablation and His bundle pacing. Europace. 2017;19:iv10-iv16.

12 Patel HJ, Pilla JJ, Polidori DJ, et al. Ten weeks of rapid ventricular pacing creates a long-term model of left ventricular dysfunction. J Thorac Cardiovasc Surg. 2017;119:834-41.

13 Hunter RJ, Berriman TJ, Diab I, et al. A randomized controlled trial of catheter ablation versus medical treatment of atrial fibrillation in heart failure (the CAMTAF trial). Circ Arrhythm Electrophysiol. 
2014;7(1):31-8.

14 Lustgarten DL, Calame S, Crespo EM, et al. Electrical resynchronization induced by direct His-bundle pacing. Heart Rhythm. 2010;7(1):15-21.

15 Boczar K, Slawuta A, Zabek A, et al. Cardiac resynchronization therapy with His bundle pacing. Pacing Clin Electrophysiol. 2019;42(3):374-380.

16 Sharma PS, Naperkowski A, Bauch TD, et al. Permanent His Bundle Pacing for Cardiac Resynchronization Therapy in Patients With Heart Failure and Right Bundle Branch Block. Circ Arrhythm Electrophysiol. 2018;11(9):e006613.

17 Nabeta T, Inomata T, Iida Y, et al. Prognostic significance of beta-blocker up-titration in conjunction with cardiac resynchronization therapy in heart failure management. Heart Vessels. 2016;31(7):1109-16.

18 Jacobsson J, Reitan C, Carlson J, et al. Atrial fibrillation incidence and impact of biventricular pacing on long-term outcome in patients with heart failure treated with cardiac resynchronization therapy. BMC Cardiovasc Disord. 2019;19(1):195.

19 Hayes DL, Boehmer JP, Day JD, et al. Cardiac resynchronization therapy and the relationship of percent biventricular pacing to symptoms and survival. Heart Rhythm. 2011;8(9):1469-75.

20 Arnold AD, Shun-Shin MJ, Keene D, et al. His resynchronization versus biventricular pacing in patients with heart failure and left bundle branch block. J Am Coll Cardiol. 2018;72(24): 3112-3122.

21 Suman-Horduna I, Roy D, Frasure-Smith N, et al. Quality of Life and Functional Capacity in Patients With Atrial Fibrillation and Congestive Heart Failure. J Am Coll Cardiol. 2013;61(4): 455-460.

22 Mukharji J, Rehr RB, Hastillo A, et al. Comparison of atrial contribution to cardiac hemodynamics in patients with normal and severely compromised cardiac function. Clin Cardio. 1990;13(9):639-43.

23 Padeletti L, Fantini F, Michelucci A, et al. Rate stabilization by right ventricular apex or His bundle pacing in patients with atrial fibrillation. Europace. 2005; 7(5): 454-9. 